Using light to probe neuronal function

This content has been downloaded from IOPscience. Please scroll down to see the full text. 2015 EPL 11138001

(http://iopscience.iop.org/0295-5075/111/3/38001)

View the table of contents for this issue, or go to the journal homepage for more

Download details:

IP Address: 150.203.159.178

This content was downloaded on 05/11/2015 at 02:29

Please note that terms and conditions apply. 


\title{
Perspective
}

\section{Using light to probe neuronal function}

\author{
Vincent R. Daria ${ }^{1(a)}$ and Hans-A. Bachor ${ }^{2}$ \\ 1 John Curtin School of Medical Research, Australian National University - Canberra, ACT, Australia \\ ${ }^{2}$ Research School of Physics and Engineering, Australian National University - Canberra, ACT, Australia
}

received 26 June 2015; accepted in final form 30 July 2015

published online 24 August 2015

\author{
PACS 87.85.D- - Applied neuroscience \\ PACS 87.85.0x - Biomedical instrumentation and transducers, including \\ micro-electro-mechanical systems (MEMS) \\ PACS 87.85.Pq-Biomedical imaging
}

\begin{abstract}
In the last few years a multi-disciplinary approach has been launched to investigate the brain using new techniques, which are capable of probing neuronal function across the entire length scales of the brain. Here, we discuss optical tools and spatial light patterning techniques to investigate brain function from the perspective of individual neurons and neuronal circuits. We discuss both biochemical and genetic tools to stimulate neurons, as well as techniques to record neuronal activity. We discuss optical projection and imaging tricks that can be dynamically customized to a particular neuron morphology and neuronal circuit layout facilitating a systematic study of their input/output transfer functions. These optical techniques will play a major role towards understanding the operation of a brain.
\end{abstract}

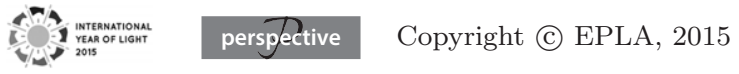

Introduction. - How individual neurons and neuronal circuits process their input to produce outputs remains one of Nature's greatest unsolved mysteries. While physicists are pushing to go beyond fundamental limits to understand basic forces of Nature, including quantum and subatomic physics, the key ingredients to probe cellular processes are within detectable length scales. Yet our understanding of the neuron's complex computational processes is still limited. Studies at extreme ends of the length scales provide complementary information. Macroscopic whole brain imaging (e.g., via magneto-resonance imaging) directs us to specific brain areas that are responsive during particular brain tasks. On the other end of the length scale, extensive studies have charted the composition of proteins in the cell membrane and processes at the molecular level. However, it remains a mystery how the complex organisation of these seemingly random molecular processes builds up a systematic computational output such as a predictable sensory response or even the simplest of "thought processes". Our level of understanding is muddled with the complex morphology of dendritic trees and the circuits they form with other neurons.

\footnotetext{
(a) E-mail: vincent.daria@anu.edu.au (corresponding author)
}

Optics can resolve individual neurons and circuits. While light scattering through optically thick mammalian brain tissues may pose as a problem for in vivo imaging, studying neuronal circuitry of simpler transparent organisms (e.g., zebrafish) may provide significant pieces of the puzzle $[1,2]$. New wavefront-engineering methods may be applied to image deep through optically thick mammalian brain. Apart from imaging, using light to probe brain function has been bolstered by recent developments in photosensitive biochemical compounds and genetically expressed proteins. Optics has now been heavily used for stimulating and recording neuronal activity, via lightsensitive actuators and reporters, to analyze the dynamics and principles of neuronal circuitry [3-6]. These developments involve the innovative use of light to facilitate accurate and minimally invasive methods to investigate brain function. Conventional electrophysiology methods lag in flexibility to design experiments that probe complex neuronal processes. Hence, dynamics and principles of neuronal circuitry will rely heavily on these optical methods to detect activity in large numbers of neurons, and to provide a means of selectively stimulating sub-sets of neurons.

The combined expertise from various fields of science could facilitate the creative design of new techniques, capable of probing neuronal function covering the entire 

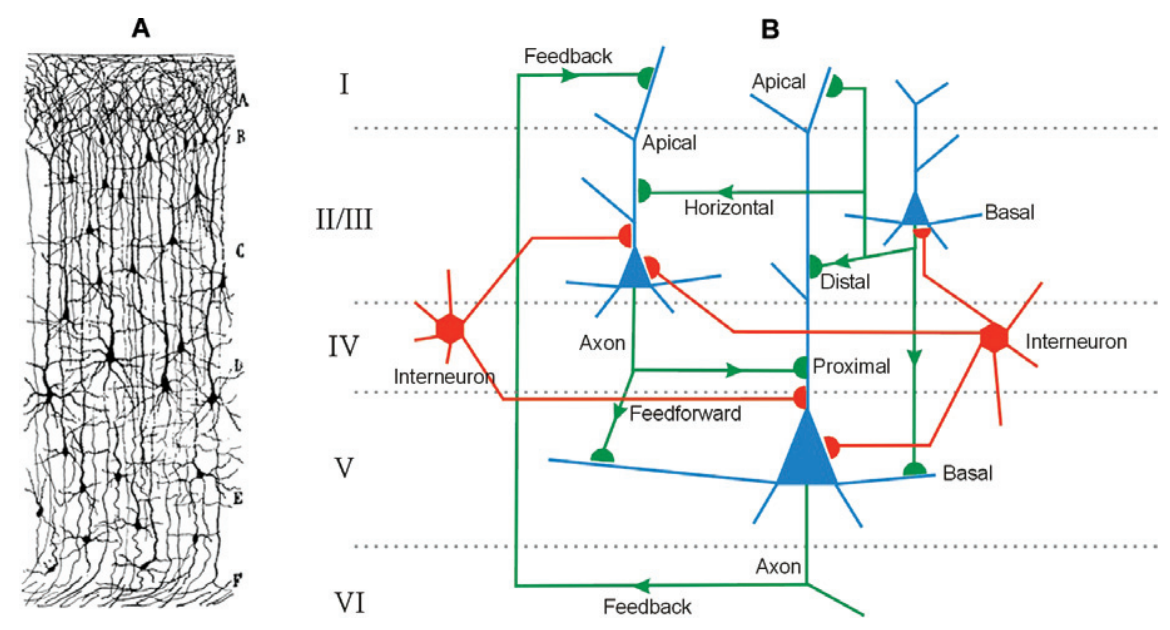

Fig. 1: (Colour on-line) (a) Illustration by S. Ramon y Cajal showing neuronal cells in the cortex. Figure taken from Cajal [10]. (b) Simplified schematic of a neuronal circuit showing connections to/from pyramidal cells in layer II/III and layer V as well as interneurons in layer IV. Colour code: blue: input/receptors; green: excitatory output; and red: inhibitory output.

length scales of brain function. Optimal use of light's properties will play a major role in this undertaking, as the big chunk of the puzzle is resolvable within optical limits for probing and visualizing neuronal activity. In this review, we will discuss various spatial light patterning techniques to understand brain function from the perspective of individual neurons and neuronal circuits. We start with discussing biochemical and genetic techniques to actuate neurons or to initiate neuron response, as well as techniques to record neuronal activity. Finally, we discuss optical projection and imaging tricks that can be dynamically customized to a particular neuronal circuit layout facilitating a systematic study of input/output transfer function.

Probing how the brain processes information. The cerebral cortex of the mammalian brain processes sensory inputs and generates motor commands among other functions. It has a laminar structure (see fig. 1(a)) populated with pyramidal cells, which are involved in higher cognitive functions [7]. Pyramidal neurons in layer II/III and layer $\mathrm{V}$ have been extensively studied morphologically [8]. These cells are characterised as having two major dendritic domains: apical and basal. The apical dendrites branch out from a single apical trunk, which extends from the apex of the soma opposite the axon. The basal dendrites, on the other hand, extend directly from the base of the soma. Cortical pyramidal neurons are broadly interconnected (see fig. 1(b)) [9]. There are feed forward connections from layer-II/III pyramidal neurons to basal dendrites and proximal apical dendrites of layer-V pyramidal neurons. There are also recurrent (feedback) connections from layer- $\mathrm{V}$ to apical dendrites of layer II/III. Horizontal connections within layer-II/III as well as within layer $\mathrm{V}$ also exist. Pyramidal neurons also respond differently depending on the location of the synapse, whether it is proximal or distal with respect to the soma. The variety of connections indicates that the various dendritic domains may play different roles in neuronal processing of its inputs.

Processing in the cerebral cortex is achieved with neurons receiving and transmitting information through the connections, otherwise known as synapses. A neuron's dendritic tree extends spatially in three dimensions (3D) and the inputs come in particular temporal sequences. Thus, each neuron receives multiple synaptic inputs in four dimensions (4D). As the neuron processes these inputs, it fires an action potential, consequently sending its processed information to other neurons in the network. When a neuron fires an action potential, excitatory neurotransmitters (e.g., glutamate) are released from the terminal boutons of the axon and bind unto receptors of a receiving neuron. Binding of a neurotransmitter opens up ion channels and results in a flow of ions into the postsynaptic cell causing the neuron to depolarize (or change in the cell's membrane potential). As the neuron receives more synaptic inputs, it depolarizes further to reach a threshold and eventually fires an action potential.

Analysing the input/output characteristics of neurons could therefore lead us to a better understanding of how information is processed in the brain [8]. Moreover, as these neurons are interconnected to form a circuit, probing multiple cells and correlating their firing of APs could elucidate the role of certain neurons during information processing. The conventional method for studying such neuronal activity is to use microelectrodes to directly measure the electrical states of a neuron as neurotransmitters transfer between neurons. Such electrodes can also be used to inject current into the neuron to activate channels and cause neurons to fire an action potential and evoke the release of neurotransmitters. These microelectrodes have also been instrumental in charting out distribution of ion-channels present in the cell membrane. 

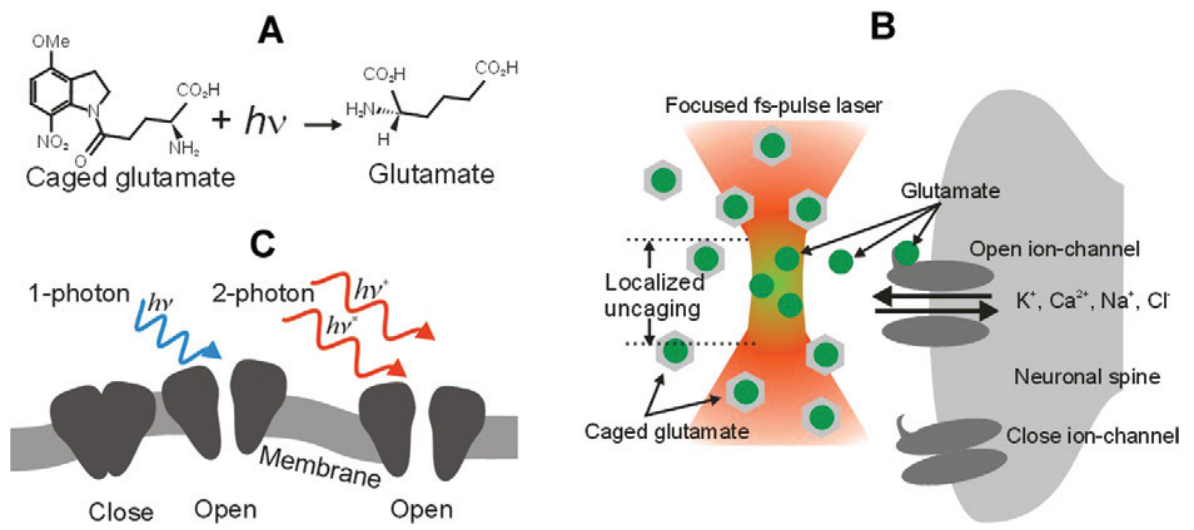

Fig. 2: (Colour on-line) (a) Photochemical reaction for glutamate uncaging. (b) Illustration of 2P uncaging near dendritic spines where neurotransmitter-gated ion channels are located. (c) Light-activated ion channels (e.g., channelrhodopsin) expressed in cell membranes. Ion channels are not drawn to scale.

Yet the use of microelectrodes is constrained especially when probing and stimulating at multiple points within the neuron and around the circuit. Microelectrode arrays can also be used, but the accuracy is limited, as the sampling points cannot be customized with neuronal morphology or with the natural circuit layout of the neurons.

With such limitations in the use of microelectrodes, a more versatile method is required to probe neuronal function. Using light to stimulate and record neuronal activity solves such difficulty. Light can non-invasively penetrate through tissues up to certain extent and patterned light can be projected to match specific neuronal morphology or circuitry. Systematic probing of synaptic inputs via controlled spatio-temporal photostimulation leading to an output response is an important step towards understanding information processing in the brain. Moreover, using light to record the neuron's response could complete an all-optical platform for analyzing neuronal activity. In the next section, we look into a variety of chemical and genetic tools to make neurons responsive to light either for stimulation and for recording neuronal activity.

Light-sensitive actuators and reporters of neuronal activity. -

Actuators. Manipulating neuronal activity with lightsensitive molecular cages has been studied for over two decades [3]. Stimulating synaptic inputs (actuator) with light can be achieved by a photochemical process where neurotransmitters are released from their chemical cage near binding sites of a receiving neuron. Light with energy $h \nu$ per photon (here $h$ is Planck's constant, $\nu$ is the frequency of light) can release the neurotransmitter from its cage. Figure 2(a) illustrates the photochemical reaction whereby a neurotransmitter (e.g., glutamate) is freed from its cage. When released, the uncaged neurotransmitter binds to receptors in the postsynaptic membrane causing a net flow of positive ions into the cell.
For most caged compounds, one ultra-violet photon is necessary to release a neurotransmitter from its molecular cage. However, one-photon excitation (1PE) does not exhibit a localized interaction in three dimensions (3D). A neuron's dendritic arbour extends in 3D and it is important to isolate the response from a localised binding site. As such, it is important to uncage neurotransmitters within a small volume near a receptor. Localized photolysis can be achieved via two-photon excitation (2PE) wherein a tightly focused femtosecond-pulse near-infrared laser brings about uncaging within a small volume at the focus (fig. 2(b)) [10,11].

Apart from using chemically caged neurotransmitters, optogenetics has emerged over the past decade as a more preferred method to manipulate neuronal activity [5]. Optogenetics makes use of genetically encoded light-sensitive proteins called opsins, which can be expressed in the cell membrane. These opsins function as light-activated ion channels (e.g., channelrhodopsin-2, ChR2) that open up and allow influx of $\mathrm{Na}^{+}$ions causing the membrane to depolarize and trigger neuronal firing. Expression of ChR2 in mammalian cells resulted in a methodological breakthrough in neuroscience [12,13]. Expressing these channels in the cell membrane can be achieved using either transfection or transgenic techniques. Light activation can be achieved by 1-photon absorption using blue light. Two-photon activation is also possible with a fs-pulse near-infrared laser [14]. Figure 2(c) illustrates opening of ChR2 using 1- and 2-photon activation. A complementary opsin, halorhodopsin (NpHR), from the halobacterium Natronomonas pharaonis, is a chloride pump that activates in response to $570 \mathrm{~nm}$ (yellow) light and serves to inhibit neuronal activity [15]. Since the excitation wavelengths of ChR2 and NpHR are different, the two can be co-expressed in the same neuron and selectively activated by light, providing bidirectional optical control of neuronal activity [16]. There are other light-sensitive ion channels whose peak activation wavelengths are red-shifted $(570 \mathrm{~nm})$ including VChR1, a channelrhodopsin homolog 

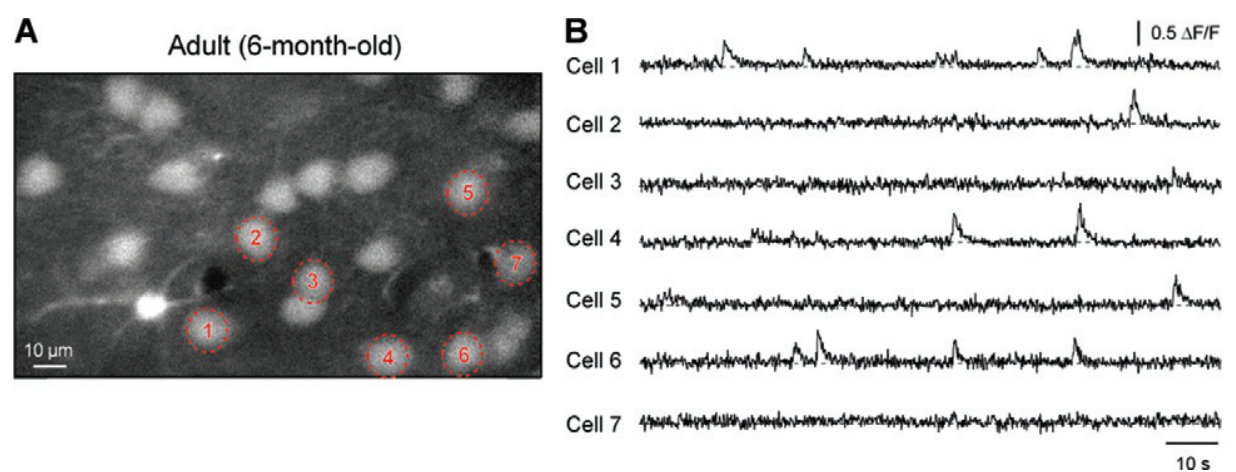

Fig. 3: (Colour on-line) In vivo $\mathrm{Ca}^{2+}$ imaging of a neuronal population. (a) Two-photon fluorescence image of L2/3 pyramidal cells in the visual cortex stained with Oregon Green BAPTA-1 (OGB-1 AM). The scale bar is $10 \mu \mathrm{m}$. (b) Spontaneous Ca ${ }^{2+}$ transients in the neurons marked in (a). Figure taken from Garaschuk L. et al. [25].

from Volvox carteri [17], and $\mathrm{C} 1 \mathrm{~V} 1_{\mathrm{T}}$, a combination of ChR1 and VChR1 $[18,19]$.

Reporters. Optical approaches to recording neuronal activity based on activity-dependent dyes have emerged. Neuronal activity is characterized by the exchange of ions to/from the neuron membrane causing the neuron to depolarize and fire action potentials. When the membrane potential of a neuron is depolarized to a certain level, voltage-gated $\mathrm{Ca}^{2+}$ channels are activated and enable $\mathrm{Ca}^{2+}$ ions to enter into the cell. Changes in the emitted fluorescence from a $\mathrm{Ca}^{2+}$ indicator dye placed in the cell could quantify the change in intracellular $\mathrm{Ca}^{2+}$ concentration and thus indirectly report neuronal firing. $\mathrm{Ca}^{2+}$ imaging of individual neurons and neuronal populations can be achieved with multi-channel image sensors such as a charge-coupled device (CCD) camera. A neuron's $\mathrm{Ca}^{2+}$ response is relatively slow $(\sim 200 \mathrm{~ms})$ so a standard camera refresh rate of $60 \mathrm{~Hz}$ is enough to capture $\mathrm{Ca}^{2+}$ dynamics from single spines to populations of neurons. In brain slices, it has been used to study $\mathrm{Ca}^{2+}$ influx into dendrites as a result of stimulation [20-22] and has helped to elucidate the existence of $\mathrm{Ca}^{2+}$ electrogenesis in the distal apical dendrite of pyramidal neurons in the rat neocortex [23]. To improve the image contrast especially when imaging thick brain slices, a single-photon (1P) confocal fluorescence microscope can be used. Depending on the scan rate, raster scanning could take longer than wide-field imaging CCD cameras but they have higher SNR. Another alternative is to use a spinning-disk confocal microscope with multiple pinholes [24]. For in vivo experiments, however, using a $2 \mathrm{P}$ microscope for $\mathrm{Ca}^{2+}$ imaging allows for deeper imaging of neuronal populations compared to $1 \mathrm{P}$ confocal microscopy (see fig. 3) [25]. This technique has been used to observe back-propagating somatic action potentials into the dendritic tree by imaging from multiple dendritic compartments at once [26-28].

Another method to record neuronal activity is via voltage-sensitive dyes (VSDs), which directly monitor changes in the membrane potential [6]. VSDs are molecules that change their fluorescent properties with changes in the membrane potential [29]. It has been used to monitor the electrical activity of single cells $[30,31]$ or neuronal populations [32-35]. VSD imaging can temporally resolve action potentials and, therefore, it is inherently faster than $\mathrm{Ca}^{2+}$ imaging.

There are also genetically encoded reporters that are calcium indicators and voltage indicators. These reporters can be genetically specified for in vivo studies. Most genetically encoded calcium indicators (GECI) used are GCaMPs and their derivatives [36-39]. GECIs have the advantage of being applicable to long-term in vivo imaging. 2P imaging of GECIs in vivo, combined with traditional stimulation techniques, has been used to monitor dendritic $\mathrm{Ca}^{2+}$ signals $[14,39]$. Genetically encoded voltage indicators (GEVIs), on the other hand, have lower signal-to-noise ratio (SNR) compared to GECIs due to the lower number of indicator molecules that can be accommodated in the cell membrane [40]. But their advantages include higher sensitivity to sub-threshold activities, including membrane hyperpolarization, and the ability to resolve higher firing frequencies compared to GECIs.

\section{Patterned illumination for stimulating and recording neuronal activity. -}

Sequential illumination (scanning). In order to effectively use these photochemical and genetic tools to study brain dynamics, we need to position the excitation light at particular regions of interest within the brain (or slice). The galvanometer mirrors (GMs) of a standard laser-scanning $2 \mathrm{P}$ microscope [41] can be used to position a single focal spot for uncaging of caged neurotransmitters along dendritic branches (see fig. 4) [42,43]. This has also been used to generate action potential with cultured ChR2-expressing hippocampal neurons by scanning the excitation spot in a spiral trajectory covering the soma [44]. Action potentials from single cells have also been initiated through $2 \mathrm{P}$ glutamate uncaging in rat brain slices by scanning the excitation spot either across 12 dendritic sites [45] or 12 sites in a circular arrangement at the soma [46]. However, electro-mechanical 
A

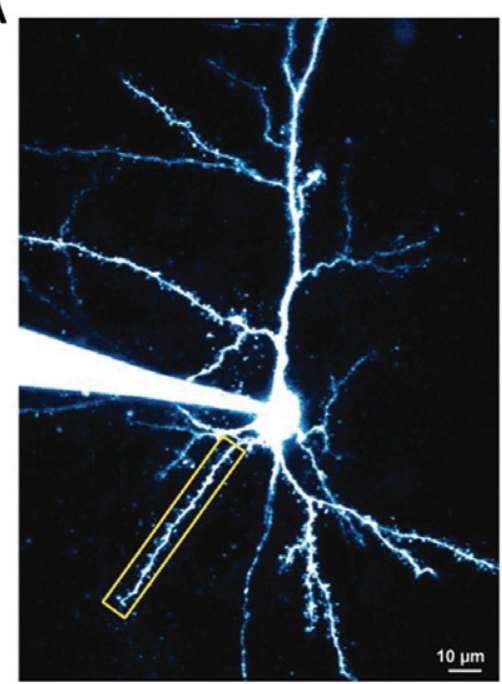

B

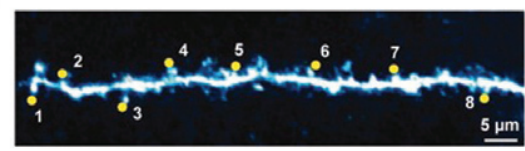

C
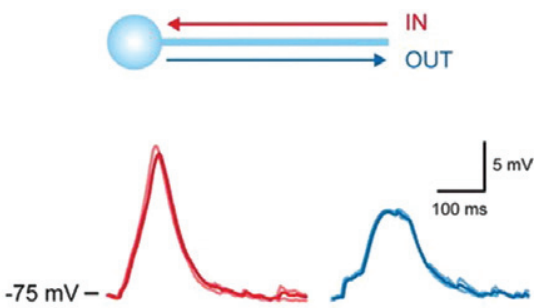

Fig. 4: (Colour on-line) Two-photon time-multiplexed multi-site uncaging. (a) Successive stimulation along a single branch (b) of a L2/3 pyramidal cell where the (c) integration for inward and outward directions is compared. Figure taken from Branco T., Clark B. A., Häusser M. [42]. The scale bar is $10 \mu \mathrm{m}$.

A

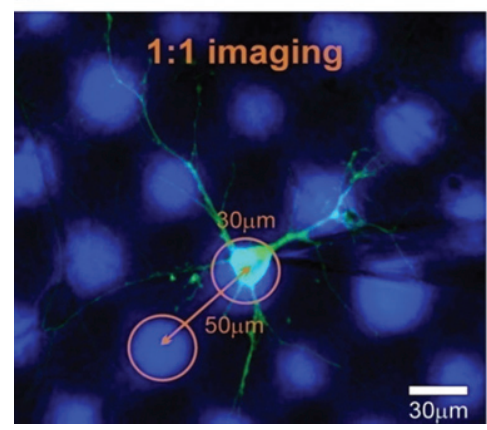

B
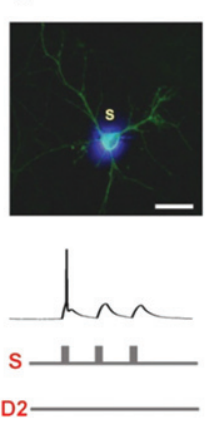

C
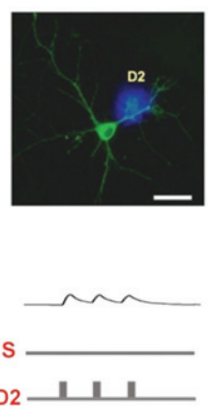

D
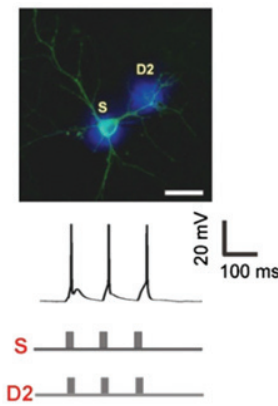

Fig. 5: (Colour on-line) Patterned excitation with a micro-LED array. (a) Bright-field image of micro-LEDs projected onto a cultured hippocampal neuron. (b) Somatic, (c) dendritic and (d) somato-dendritic excitation. Somatic voltage responses due to ChR2-evoked input currents and illumination pulses are shown. The scale bar is $30 \mu \mathrm{m}$. Figure taken from Grossman N. et al. [51].

beam scanners (e.g., GMs) are slow compared to using acousto-optic deflectors (AODs) $[47,48]$. AODs deflect an incident beam via a controllable optical grating, which is formed by standing waves induced from an applied radiofrequency signal. A combination of four AODs can be used to position the spot in $3 \mathrm{D}$ where high-speed volumetric imaging of neuronal activity can be performed $[49,50]$.

Parallel illumination. Compared to scanning, patterned parallel illumination allows for simultaneous excitation at multiple locations. The availability of programmable optical devices such as light-emitting diode (LED) arrays as well as amplitude and phase-only spatial light modulators (SLMs) has allowed the use of patterned illumination techniques. Micro-LED arrays have been used for patterned stimulation with high spatiotemporal resolution (fig. 5) [51]. Such an array was used to evoke action potentials in individual ChR2-expressing cultured hippocampal neurons and ganglion cells in mouse ChR2-transfected retina [51]. It was also used to excite somatic (fig. 5(b)), dendritic (fig. 5(c)) and somato-dendritic (fig. 5(d)) segments simultaneously.

Another way to modulate the amplitude is to use a digital micro-mirror device (DMD), which is an array of independently controllable micro-mirrors. When the DMD is placed at the image conjugate of the neuronal tissue, each micro-mirror could reflect light that corresponds to a photostimulation site. DMDs can achieve switching rates up to $22 \mathrm{kHz}$, allowing spatiotemporal control of excitation patterns. Photostimulation with a DMD system has been used to stimulate two LiGluR-expressing cells in a rat hippocampal culture simultaneously [52]. It has been used to activate ChR2-expressing glomeruli to map glomerular inputs to individual mitral/tufted cells in the olfactory bulb [53]. It has also been used for UV photolysis of caged glutamate along multiple dendritic 

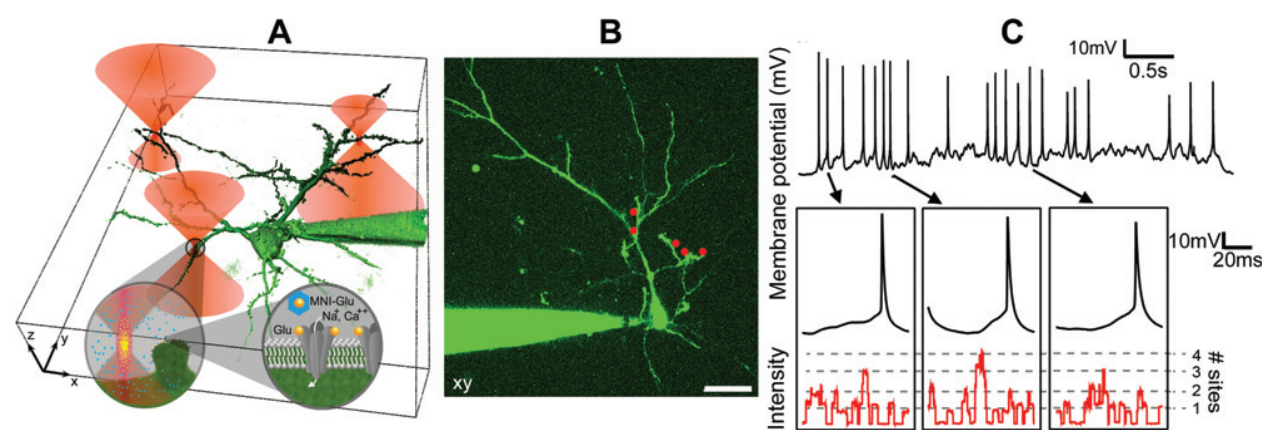

Fig. 6: (Colour on-line) (a) Holographic projection using a programmable spatial light modulator generates multiple foci for uncaging of caged neurotransmitter (e.g., MNI-Glu) along a neuron's dendritic arbour in 3D. The released neurotransmitters (Glu) bind to ionotropic ligand-gated ion channels causing an excitatory post-synaptic current flow of $\mathrm{Na}^{+} / \mathrm{Ca}^{++}$into the neuron. Figure taken from Go M. A. et al. [63]. (b) Layer-II/III pyramidal neuron with five uncaging sites along apical oblique branches. The scale bar is $25 \mu \mathrm{m}$. (c) Representative membrane potential time courses showing the generation of action potentials when at least three sites are simultaneously incident along the uncaging sites in (b). Panels (b) and (c) are revised from Go M. A. et al. [64].

branches to study between-branch summation [54] and to simultaneously activate a large group of neurons distributed across multiple cortical regions [55].

Another alternative for generating patterned light is the generalised phase contrast (GPC) method that uses a phase-only SLM together with a phase contrast filter (PCF) [56]. The GPC method is a phase-to-intensity converter where the spatial intensity pattern at the output is a conjugate image of the spatial phase pattern at the input. Such technique can be effectively used for 1P-patterned excitation. However, for $2 \mathrm{P}$ excitation, the GPC method can be combined with temporal focusing and has been applied to stimulate ChR2 [57]. The principle of temporal focusing is to spatially separate the spectral components of the input laser beam with a diffraction grating and then recombine them only at the focal plane $[58,59]$. Dispersion broadens the pulse before and after the focal plane and hence limits non-linear $2 \mathrm{P}$ excitation only at the focal plane. The $2 \mathrm{P}$ excitation probability varies inversely as the square of the pulse width. This allows an axial resolution close to that of confocal and $2 \mathrm{P}$ excitation while covering a wide excitation area rather than a single diffraction-limited spot [60].

Phase-only SLMs can also be used with the holographic projection technique where a computer-generated phase hologram produces reconfigurable patterned illumination in $3 \mathrm{D}$. This technique has been demonstrated for 1PE [61] and $2 \mathrm{PE}$ [62-64] patterned uncaging of neurotransmitters in a plane. The holographic technique has also been used to simultaneously induce arbitrary synaptic inputs in 3D [63]. Figure 6(a) illustrates how the holographic method is used to produce multiple spatially distinct foci projected along the 3D dendritic arbor of a neuron. Three foci positioned at different planes are shown where all foci simultaneously induce uncaging of neurotransmitters. A DMD can be placed at the projection plane where each focus is temporally gated with micro-mirrors [64]. With high-speed switching, the stimulation foci can be switched rapidly within physiological time scales therefore emulating how a neuron receives multiple synaptic inputs from neighboring neurons. Figure 6(b) shows uncaging along the apical oblique dendrites of a layer-II/III neuron where the neuron fires action potentials when at least three (3) uncaging sites are simultaneously active (fig. 6(c)). Aside from photostimulation, the holographic projection technique has also been used to simultaneously image neuronal activity within dendrites via VSD imaging [65] and from neuronal populations via calcium imaging $[62,66,67]$.

Summary. - Optics has become a very attractive tool for analysing neuronal information processing. In combination with photosensitive biochemical and genetically encoded proteins, light's dynamic properties and capacity to penetrate through biological tissues could allow for an accurate and comprehensive study of neuronal communications. In parallel to imaging, laser-based stimulation is now an established technique for studying neuronal function. In vivo experiments will benefit as deeper sections of the intact brain become accessible for systematic behavioural studies with an accuracy of analysis down to the level of single-neuron response. This analysis of the information processes in the brain offers overwhelming benefits ranging from fundamental questions, including the understanding of brain development, to medical applications, such as a better understanding of mental disorders.

\section{REFERENCES}

[1] Ahrens M. et al., Nature, 485 (2012) 471.

[2] Ahrens M. et al., Nat. Methods, 10 (2013) 413.

[3] Callaway E. and Katz L., Proc. Natl. Acad. Sci. U.S.A., 90 (1993) 7661.

[4] Svoboda K. et al., Nature, 385 (1997) 161.

[5] Nagel G. et al., Science, 296 (2002) 2395.

[6] Grinvald A. and Hildesheim R., Nat. Rev. Neurosci., 5 (2004) 874. 
[7] Cajal S. R., Histology of the Nervous System of Man and Vertebrates (Oxford University Press) 1995.

[8] Spruston N., Nat. Rev. Neurosci., 9 (2008) 206.

[9] Thomson A. et al., Cerebral Cortex, 12 (2002) 936.

[10] Denk W., Proc. Natl. Acad. Sci. U.S.A., 91 (1994) 6629.

[11] Matsuzaki M., Ellis-Davies G. C. R. and Kasai H., J. Neurophysiol., 99 (2008) 1535.

[12] Nagel G. et al., Proc. Natl. Acad. Sci. U.S.A., 100 (2003) 13940.

[13] Boyden E. S. et al., Nat. Neurosci., 8 (2005) 1263.

[14] Tian L. et al., Nat. Methods, 6 (2009) 875.

[15] Zhang F. et al., Nature, 446 (2007) 633.

[16] Han X. and Boyden E. S., PLoS ONE, 2 (2007) e299.

[17] Zhang F. et al., Nat. Neurosci., 11 (2008) 631.

[18] PaCker A. M. et al., Nat. Methods, 9 (2012) 1202.

[19] Prakash R. et al., Nat. Methods, 9 (2012) 1171.

[20] TANK D. W. et al., Science, 242 (1988) 773.

[21] Yuste R. and Katz L. C., Neuron, 6 (1991) 333.

[22] Yuste R. et al., Neuron, 13 (1994) 23.

[23] Larkum M. E., Kaiser K. M. M. and Sakmann B., Proc. Natl. Acad. Sci. U.S.A., 96 (1999) 14600.

[24] Namiki S. and Ikegaya Y., Biol. Pharm. Bull., 32 (2009) 1.

[25] Garaschuk O., Milos R.-I. and Konnerth A., Nat. Protocols, 1 (2006) 380.

[26] Svoboda K. et al., Nature, 385 (1997) 161.

[27] Helmchen F. et al., Nat. Neurosci., 2 (1999) 989.

[28] Waters J. and Helmchen F., J. Neurosci., 24 (2004) 11127.

[29] Chemla S. and Chavane F., J. Physiol., 104 (2010) 40.

[30] Salzberg B. M., Davila H. V. and Cohen L. B., Nature, 246 (1973) 508.

[31] Cohen L. et al., J. Membr. Biol., 19 (1974) 1.

[32] Baker B. J. et al., Cell Mol. Neurobiol., 25 (2005) 245.

[33] Kuhn B., Denk W. and Bruno R. M., Proc. Natl. Acad. Sci. U.S.A., 105 (2008) 7588.

[34] Brown C. E. et al., J. Neurosci., 29 (2009) 1719.

[35] XU X. et al., J. Neurophys., 103 (2010) 2301.

[36] Nakai J., Ohkura M. and Imoto K., Nat. Biotechnol., 19 (2001) 137.
[37] Nagai T. et al., Proc. Natl. Acad. Sci. U.S.A., 98 (2001) 3197.

[38] Hasan M. T. et al., PLoS Biol., 2 (2004) 763.

[39] Heim N. et al., Nat. Methods, 4 (2007) 127.

[40] Knöpfel T., Nat. Rev. Neurosci., 13 (2012) 687.

[41] Denk W., Strickler J. H. and Webb W. W., Science, 248 (1990) 73.

[42] Branco T., Clark B. A. and Häusser M., Science, 329 (2010) 1671.

[43] Branco T. and Häusser M., Neuron, 69 (2011) 885.

[44] Rickgauer J. and Tank D., Proc. Natl. Acad. Sci. U.S.A., 106 (2009) 15025.

[45] Kantevari S. et al., Nat. Methods, 7 (2010) 123.

[46] Nikolenko V., Poskanzer K. E. and Yuste R., Nat. Methods, 4 (2007) 943.

[47] Saggau P., Bullen A. and Patel S. S., Cell Mol. Biol., 44 (1998) 827.

[48] Shoham S. et al., Nat. Methods, 2 (2005) 837.

[49] Reddy G. D. et al., Nat. Neurosci., 11 (2008) 713.

[50] Katona G. et al., Nat. Methods, 9 (2012) 201.

[51] Grossman N. et al., J. Neural Eng., 7 (2010) 016004.

[52] Wang S. et al., Nano Lett., 7 (2007) 3859.

[53] Dhawale A. K., Nat. Neurosci., 13 (2010) 1404.

[54] Liang C. W. et al., J. Vis. Exp., 49 (2011) e2003.

[55] Jerome J. et al., Front. Syst. Neurosci., 5 (2011) 70.

[56] Glückstad J., Opt. Commun., 130 (1996) 225.

[57] Papagiakoumou E. et al., Nat. Methods, 7 (2010) 848.

[58] Zhu G. et al., Opt. Exp., 13 (2005) 2153.

[59] Oron D., Tal E. and Silberberg Y., Opt. Exp., 13 (2005) 1468.

[60] Andrásfalvy B. K. et al., Proc. Natl. Acad. Sci. U.S.A., 107 (2010) 11981.

[61] Lutz C. et al., Nat. Methods, 5 (2008) 821.

[62] Nikolenko V. et al., Front. Neural Circuits, 2 (2008) 5.

[63] Go M. A. et al., J. Biophoton., 5 (2012) 745.

[64] Go M. A. et al., Front. Cell. Neurosci., 7 (2013) 231.

[65] Foust A. et al., Neurophotonics, 2 (2015) 21007.

[66] Ducros M. et al., Proc. Natl. Acad. Sci. U.S.A., 110 (2013) 13138.

[67] Quirin S. et al., Front. Neural Circuits, 8 (2014) 29. 